may occasion surprise that the choice of treatment should be left to the dentists, who were far from unanimous in their replies to the question "Do you ask about heart murmurs and rheumatic fever when a new patient presents ?" Should it not be the duty of a physician in charge of a patient with a rheumatic heart lesion to supervise all such treatment?

Durack's own strong views are based not so much on clinical experience (which cannot be so extensive as to afford statistical proof of preventive efficacy) as on experimental study. When catheters are fixed in the right atrium and left ventricle of rabbits via the jugular vein and carotid artery small sterile vegetations form on the valves on which these impinge. A suspension of Strep. viridans may then be injected intravenously and antibiotic treatment given. After 24 hours the animals may be killed and quantitative cultures made from the vegetations. In their main series of such experiments Durack and Petersdorf ${ }^{2}$ found that penicillin sterilized the vegetations only when a high initial level was maintained for nine hoursas is done by a large intramuscular dose of procaine penicillin. The best results were given by a combination of penicillin and streptomycin or of ampicillin and streptomycin, and by vancomycin. Tetracycline, erythromycin, cephalexin, clindamycin, rifampicin, and cotrimoxazole were ineffective. Doubtless in view of spurious claims which have been made for tetracycline Southwick and Durack ${ }^{3}$ tried continued treatment of the experimental infection with this antibiotic for seven days; they found that streptococci still survived in the vegetations. Hence the final recommendation made in Durack's paper on the practice among dentists is that prophylaxis should be either with vancomycin ( $1 \mathrm{~g})$ or with a combination of penicillin 2 mega units, procaine penicillin 500000 units, and streptomycin $1 \mathrm{~g}$; treatment should be given as a single injection shortly before extraction.

Adequate and sufficiently sustained blood levels for a fully dependable effect can be achieved only by parenteral therapy, yet only $12 \%$ of dentists listed this as first choice. The question that nevertheless remains is whether the treatment needs to be as arduous as that proposed. Vancomycin has to be given intravenously, which must detract from its popularity. And is the large dose of penicillin combined with streptomycin really necessary? Is it necessary to give streptomycin at all, unless in a patient already on penicillin treatment, whose mouth streptococci will then be resistant?

One very peculiar case was reported by Durack and Littler, 4 in which bacterial endocarditis was believed to have developed immediately despite two days' cover with generous doses of penicillin and cloxacillin. As pointed out in a subsequent letter 5 "three days is an incredibly short time for endocarditis to appear after dental extraction," and the presumption is that it already existed before the operation. In the main experimental study already quoted a large dose of procaine penicillin alone was effective. Those who have hitherto relied on a single full dose of fortified procaine penicillin may be content still to do so despite these counsels of perfection. Even though the conclusions drawn from it may seem extreme, Durack's experimental study of the problem is much to be commended, and others may be encouraged to emulate it; a modified technique might either confirm or qualify the conclusions drawn and possibly render the clinician's taks less burdensome.

1 Durack, D. T., British Heart fournal, 1975, 37, 478

2 Durack, D. 1., and Petersdorf, R. G., Fournal of Clinical Investigation, $1973,52,592$.

Southwick, F. S., and Durack, D. T., fournal of Clinical Pathology, 1974, $27,261$.

Durack, D. T., and Littler, W. A., Lancet, 1974, 2, 846.

5 Fleming, H. A., Lancet, 1974, 2, 1078.

\section{Pleural Effusion}

Pleural effusion may occur from transudation or exudation. Pleural transudates are clear watery fluids, low in protein, whereas pleural exudates are yellowish and may contain blood and cells. They often clot spontaneously on aspiration and have a higher protein content $(>3 \mathrm{~g} / \mathrm{l})$.

Very small effusions can be seen only on radiological examination by obliteration of the normal costophrenic angle when the patient is in the erect position. Probably there has to be $500 \mathrm{ml}$ or more of fluid before its presence can be detected reliably on physical examination. By far the most important physical sign is stony dullness on percussion over the fluid, and this is usually associated with absent or reduced tactile vocal fremitus and reduced breath sounds, though above or medial to the effusion bronchial breathing is often heard. Vocal resonance may become aegophonic-a bleating quality -but only in the larger effusions is the mediastinum displaced to the opposite side, and this sign may be absent even in large effusions if there is underlying collapse of the lung due, for instance, to a bronchial neoplasm.

Pleural transudates are frequently bilateral and are due to a hydrodynamic imbalance of the forces maintaining capillary blood pressure. They are usually easily diagnosed; there is generally clear clinical evidence of the primary disorder, such as congestive cardiac failure, nephrotic syndrome, hepatic cirrhosis, or malnutrition. A very rare cause of pleural transudates is Meigs's syndrome, ${ }^{1}$ in which usually bilateral effusions are associated with ascites and an ovarian tumour. The collection of fluid in the serous spaces may clear when the tumour is removed; the mechanism is not understood.

Pleural exudates usually occur as a result of primary disease of the underlying lung or pleura. Sometimes pleural transudates and exudates may coexist, and after a patient with congestive cardiac failure and bilateral pleural effusions has responded to therapy one pleural effusion may persist and be found to be due to unsuspected secondary pleural malignant disease. Neoplastic disease is, indeed, among the common causes of pleural exudates, particularly primary carcinoma of the bronchus, of which an effusion may be the earliest physical sign. Pleural exudates also occur due to pleural mesothelioma or metastatic pleural spread from a primary growth elsewhere in the body. Pleural exudates commonly complicate bacterial pneumonia, especially when it has been treated inadequately with antibiotics. Pleural exudates also occur in pulmonary tuberculosis, after pulmonary infarction, and in connective tissue diseases such as rheumatoid arthritis or systemic lupus erythematosus.

It must always be remembered that pleural effusion, especially on the right side, may arise as a result of disease below the diaphragm, such as subphrenic abscess, hepatic amoebiasis, or pyogenic hepatic abscess, and after acute pancreatitis.

Rarer causes of pleural exudates have been described: pleural effusion may be found with primary lymphoedema and yellow discoloration of the nails, the yellow nail syndrome, ${ }^{2}$ and recently pleural exudates, often blood stained, have been reported in patients on chronic dialysis for chronic renal failure. $^{3}$

The cause of most pleural effusions is easily discerned. After assessment of the clinical features and examination of the chest the sputum should be searched for organisms and malignant cells, and a sample of pleural fluid examined for blood, organisms, and cells. Malignant effusions are usually blood stained and accumulate rapidly after aspiration; 
malignant cells may be found. Culture of the pleural fluid for tubercle bacilli is essential. Tuberculous effusions usually contain large numbers of lymphocytes, while numerous polymorphs with lymphocytes point to a postinfective pleural effusion, invariably sterile on culture. Lung scans, repeated if necessary, and phlebography of the legs will help to distinguish effusion complicating pulmonary infarction. Chylous pleural effusions appear milky and are due to perforation of the thoracic duct or its malignant infiltration. Though the cause of most pleural effusions can be diagnosed by these means, in difficult cases bronchoscopy, pleural biopsy, or mediastinoscopy with biopsy may be needed.

${ }^{1}$ Meigs, J. V., American fournal of Obstetrics and Gynecology, 1954, 67, 962.

2 Hiller, E., Rosenow, E. C., and Olsen, A. M. Chest, 1972, 61, 452.

3 Berger, H. W., et al., Annals of Internal Medicine, 1975, 82, 362.

\section{Wandering Gall Bladders}

Congenital anomalies of the gall bladder, its ducts, and its blood supply are sufficiently common for the surgeon to need to be aware of these vagaries. Indeed most postoperative complications of cholecystectomy may be related to a combination of inadequate anatomical knowledge and poor visualization of the operative field. ${ }^{1}$

The gall bladder may be absent, double, abnormally shaped or malpositioned. Complete absence is rare: more often the organ is represented by a rudiment of a fibrous nodule. If it is any compensation to the patient, he might be told that the rat has no gall bladder but this does not prevent its ability to digest an extraordinary range of substances.

A double gall bladder is common in cats $(12 \%)$ and to a lesser extent in pigs, sheep, and cows, but it is rare in man. There may be two entirely separate gall bladders and cystic ducts or the gall bladder may be Y-shaped or divided by a septum. ${ }^{2}$ In such cases only one of the two gall bladders may be the site of the disease. The diagnosis may be difficult, because the apparently perfectly normal cholecystogram merely demonstrates the normal twin, whereas the second, inflamed, gall bladder is non-functioning and is not visualized. In other more fortunate instances gall stones can be seen within one loculus.

Malformations of the gall bladder are usually acquired, the viscus being kinked or else distorted into a dumb-bell or hourglass shape by inflammatory adhesions and scar tissue from repeated inflammatory episodes. A common congenital malformation seen on cholecystography or at operation or necropsy is a marked kink between the body and fundus, which produces the phrygian cap deformity-this was the liberty-cap headgear adopted by the Parisian mob at the time of the French Revolution. The deformity itself is of no clinical significance.

Anomalous positions of the gall bladder are rare. It may be buried partially or completely within the substance of the liver which may make cholecystectomy more than usually difficult. In other rare cases the gall bladder lies under the left lobe of the liver, or is placed transversely, or points posteriorly beneath the right lobe. In congenital transposition of the viscera the gall bladder and the liver may be situated in the left upper quadrant of the abdomen, and this may be associated with transposition of the heart and great vessels. Indeed, the diagnosis may initially be suggested clinically by discover- ing that the apex beat of the heart is situated on the right side. It is surprising how confused the surgeon feels when called upon to perform a left-sided cholecystectomy.

The opposite of an intrahepatic gall bladder is the ptosed or floating gall bladder, which instead of being plastered firmly against its bed in the liver is suspended from it by a mesentery which is either complete or else surrounds the fundus and body. In rare cases the gall bladder is completely invested in peritoneum and has no mesentery whatsoever. There has been one example where such a gall bladder herniated through the foramen of Winslow. Ptosis of the gall bladder is not unusual; it occurs in some $5 \%$ of individuals. Its importance is that it allows torsion of the gall bladder to occur with consequent gangrene of the organ. ${ }^{3}$

Chiavarini and his colleagues ${ }^{4}$ have recently reported an unusual example of a hypermobile gall bladder and liver. This was a 22-year-old male who gave a history of almost daily episodes of abdominal pain dating back to early childhood. A chest $x$-ray film showed a large collection of right upper quadrant gas beneath the right hemidiaphragm. A cholecystogram showed a normal gall bladder in the left upper quadrant, which subsequently gradually shifted to the right side. Barium studies showed that the stomach, which initially presented to the right of the midline, later occupied a more normal position on the left. Initial radiographs failed to show the normal liver density, but subsequently this organ was seen quite prominently in its normal position. The transverse colon was markedly dilated. At operation the liver was found to be suspended only by the falciform ligament, which was elongated and midline. The omenta were elongated, and the entire colon was quite mobile on a mesentery. The spleen, duodenum, and small intestine had normal attachments. A total abdominal visceropexy was performed with relief of symptoms. The authors were able to find only three similar cases in previous publications, and all were in men under the age of 30 . From time to time surgeons have recorded a large mobile mass within the abdomen which at laparotomy is readily delivered into the wound on its long mesentery. Fortunately, it has been possible to identify this mass as a ptosed liver before performing what would have been an all too easy inadvertent total hepatectomy.

1 Maingot, R., Abdominal Operations, 6th edn., vol. 1, p. 928. New York, Appleton, Century, Crofts, 1974

2 Gross, R. E., Archives of Surgery, 1936, 32, 131.

${ }^{3}$ Levene, A., British fournal of Surgery, 1958, 45, 338.

${ }^{4}$ Chiavarini, R. L., Chang, S. F., and Westerfield, J. D., Diagnostic Radiology, 1975, 115, 47

\section{Acute Muscle Compartment Compression Syndromes}

Volkmann's ischaemic contracture is one of the eponyms that all medical students remember, and while they may not appreciate the many ways in which the flexor muscles of the forearm can become ischaemic they all recognize the common end result-fibrosed contracted muscles and a claw hand. Yet despite this widespread appreciation of the existence and cause of Volkmann's contracture the effect of muscle ischaemia in other fascial compartments, particularly in the leg, is less well known and often undiagnosed and mistreated, ${ }^{1}$

The anterior group of muscles in the lower leg is contained 